\title{
Monte Carlo Study of Chain Entanglements in Polymer Melt
}

\author{
A. Sikorski And P. Romiszowski* \\ Department of Chemistry, University of Warsaw \\ Pasteura 1, 02-093 Warsaw, Poland
}

(Received July 6, 2001)

\begin{abstract}
The new multibond algorithm for the Monte Carlo simulations of polymers developed for an isolated chain was extended for the case of dense polymer melts. Polymer chains were confined to a simple cubic lattice with excluded volume and no attractive interactions (good solvent conditions). The simulations were carried out by the means of the classical Metropolis scheme. The algorithm was verified by the analysis of static and dynamic properties of polymer melts. The dependence of the longest relaxation time and the self-diffusion coefficient on the chain length and the polymer concentration was discussed and the proper scaling laws were formulated. The number of entanglements, their distribution, and lifetimes were determined for different chain lengths and melt concentrations using the new algorithm.
\end{abstract}

PACS numbers: $02.50 . \mathrm{Ng}, 61.25 . \mathrm{Hq}$

\section{Introduction}

The dense polymer melt is not only an interesting theoretical many-body problem but it is also of practical importance (rheology, adhesion, lubrication) [1]. The most popular model of the chain motion is the idea of reptation, which originated from the de Gennes' model [2, 3]. In this model it is assumed that chains surrounding a given chain can be treated as fixed in space and thus the motion of test chain is like a slithering snake in a tube of constraints. Predictions of this model concerning the dynamic behavior of the melt are almost the same as experimental results: in both cases the diffusion coefficient scales as $D \sim N^{-1}$ for shorter chains and as $N^{-2}$ for longer chains. For shorter chains the scaling of

*corresponding author; e-mail: prom@chem.uw.edu.pl 
viscosity is predicted correctly $\left(\eta \sim N^{1}\right)$ but for longer chains reptation theory predictions are $N^{3}$ instead of $N^{3.4}$. Computer simulation carried out for different models confirmed the experimental results [4-7]. Thus, many attempts were made in order to improve these predictions by modifying the reptation theory or by introducing different mechanisms of chain motion $[1,4,8]$.

Computer simulation is a very useful tool in studying the properties of polymer systems because real experiments cannot answer the question concerning the mechanism of chain motion in melt $[1,4]$. Even very simple lattice models in spite of some limitations appeared very useful to study dense polymer systems. Regardless of the assumed model of chain motion in the melt the question of the entanglements remains crucial. The questions arise what is the mobility of entanglements, their distribution, and lifetimes. Recently, there have been some attempts of determining the properties of polymer-polymer contacts and chain entanglements on a molecular level using the computer simulations but to date they were carried out for isolated polymers only $[9,10]$. The extensive studies of polymer systems having different internal architecture (star-branched chains, rings) showed the influence of the chain topology on its structure and dynamic properties [10-14].

Recently, we have introduced a new multibond algorithm for the Monte Carlo simulation of chain molecules that uses the asymmetric Metropolis scheme [15]. This algorithm was capable to reproduce most of features of the model of an isolated polymer chain. The extension of the algorithm can also be used for studying the properties of dense polymer melts. What is interesting, it appeared that some modifications of this algorithm applied for dense multichain systems could give some insight into the nature of entanglements. The purpose of this work is to check the validity and usability of the new algorithm for dense polymer melts and to study the nature of chain entanglements.

\section{The model and the algorithm}

The simulations were carried out for the simple model of a dense polymer melt. Chains were confined to a simple cubic lattice, i.e. every polymer segment was placed in lattice vertex $[4,5,12]$. The double occupancy of lattice points was forbidden which implied that the excluded volume effect was taken into account. No temperature dependent long-range interactions were introduced, i.e., the melt was athermal which corresponds to good solvent conditions.

In the classical simulation algorithm the conformational space was sampled by local chain micromodifications (2 or 3 polymer bonds were involved in every micromodification) [4, 14]. We have constructed a new algorithm in which larger parts of a chain can be shifted at once. The multibond algorithm was tested for single chains and reproduced proper static and dynamic properties of the system [15]. It was also shown that this algorithm was considerably faster than the older one. The multibond algorithm was recently described in detail [15] and thus we are giving only a short description here. In the new algorithm the elementary motions 
modify the large portion of the polymer chain. In the version of the algorithm presented here 6 subsequent (along the chain contour) polymer bonds undergo simultaneous modification. For such a shift of large parts of a chain there is a possibility of topological obstacles because the moved part can cross other parts of the chain or other chains. Thus, every attempt of motion is accepted after the check if points, which can be potentially occupied by a fragment of the same or other chain, are empty. In this motion the connectivity of the chain and the geometry of the lattice has to be maintained, too. Chain ends were subjected to the same 6 -bond micromodifications but the new conformation was chosen randomly. In this algorithm we can as usual define time unit which corresponded to one attempt of chain modification per one polymer bead. The polymer melt was generated and equilibrated using $10^{5}-10^{6}$ time units while the production run consisted of order of $10^{6}-10^{7}$ time units.

The systems of linear $M$ chains, each consisting of $N$ beads (or $N-1$ segments), were placed in a Monte Carlo box with periodic boundary conditions. The edge of the box was chosen large enough to avoid a chain to interact with its image or $L$ had to be greater than root-mean-square end-to-end vector $\left\langle R^{2}\right\rangle^{1 / 2}$, i.e. $L=20$ for shorter chains and $L=40$ for longer chains. The density of the melt can be defined as the ratio of the total number of polymer beads to the number of beads in the Monte Carlo box, i.e., $\varphi=M \cdot N / L^{3}$. The simulations were carried out for the chains with $N=50,100,200,400$, and 800 beads for the density $\varphi=0.5$. We also carried out simulations for systems containing chains with $N=100$ beads at different densities: $\varphi$ varied from 0.1 to 0.8 (for densities higher than 0.6 this algorithm becomes inefficient).

\section{Results and discussion}

Some parameters describing the size of a polymer chain were calculated during the simulation. These were mean-square end-to-end distance $\left\langle R^{2}\right\rangle$ and the mean-square radius of gyration $\left\langle S^{2}\right\rangle$. The values obtained are very close to those from the simulations carried out by the classical algorithm. As an example we present Fig. 1 where one can observe the dependence of $\left\langle R^{2}\right\rangle$ and $\left\langle S^{2}\right\rangle$ on the chain length $N-1$ for moderate density $(\varphi=0.5) \cdot\left\langle R^{2}\right\rangle$ and $\left\langle S^{2}\right\rangle$ scale with the chain length as $(N-1)^{1.030 \pm 0.02}$ and $(N-1)^{1.040 \pm 0.02}$, respectively. The exponents are very close to previous simulation results obtained for the same model at the same conditions by means of the classical Monte Carlo algorithm, where $\gamma_{R}=0.99$ and $\gamma_{S}=1.02[5]$ and to the theoretical predictions where $\gamma_{R}=\gamma_{S}=1$ $[1,3,4]$. The values of scaling exponents close to random flight chain (with no excluded volume) can be explained by the fact of mutual interpenetration of polymer chains $[1,4,8]$. The ratio $\left\langle S^{2}\right\rangle /\left\langle R^{2}\right\rangle$ changes from 0.164 for systems containing short chains $(N=50)$ to 0.166 for longer chains $(N=800)$ which was expected, since for random flight chain it takes the value 0.167 . The next figure (Fig. 2) presents the dependence of $\left\langle S^{2}\right\rangle$ on the density $\varphi$ for melt consisting of chains with $N=100$ beads. The size of a single polymer decreases with the increase in 


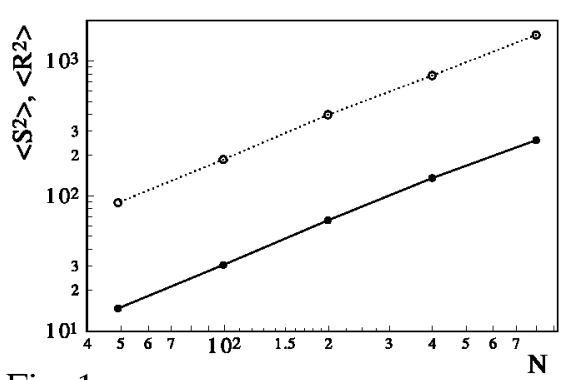

Fig. 1

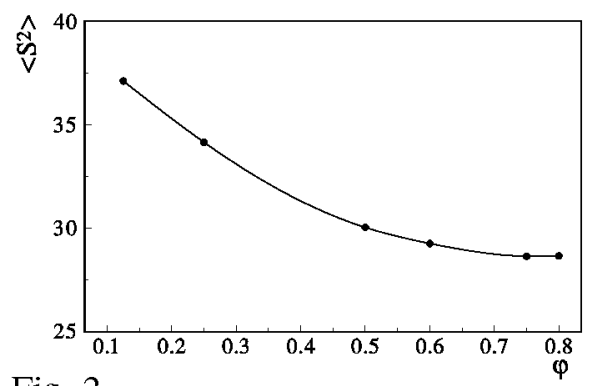

Fig. 2

Fig. 1. The mean-square end-to-end distance $\left\langle R^{2}\right\rangle$ (open circles, dotted line) and the mean-square radius of gyration $\left\langle S^{2}\right\rangle$ (solid circles, solid line) versus the chain length $N$. The case of the density $\varphi=0.5$.

Fig. 2. The mean-square radius of gyration $\left\langle S^{2}\right\rangle$ versus the density $\varphi$. The case of the chain with $N=100$ beads.

the density rather fast for $\varphi<0.5$ because chains become to interpenetrate one another. For higher densities the decrease in the polymer chain becomes slower.

The dynamic properties of the systems studied can be determined for short-time and long-time timescale. Fast processes of the chain are usually described by the longest relaxation time, which describes the process of internal rearrangements. The longest relaxation time $\tau_{\mathbf{R}}$ can be extracted from the end-to-end vector autocorrelation function as was previously described many times [4, 12, 14]. Figure 3 presents the values of $\tau_{\mathrm{R}}$ for melts at the density $\varphi=0.5$ versus the number of segments in the polymer chain $N-1$. The relaxation times scale with the chain length as $(N-1)^{2.53 \pm 0.06}$, which is very close to the results obtained for similar model using the classical algorithm where $\tau_{\mathrm{R}} \sim(N-1)^{2.63}[5,14]$. The simplest theory of Rouse (isolated chain, no hydrodynamic interactions) predict the exponent equal to $2[1,3,16]$. Experiments concerning dense polymer melts give the

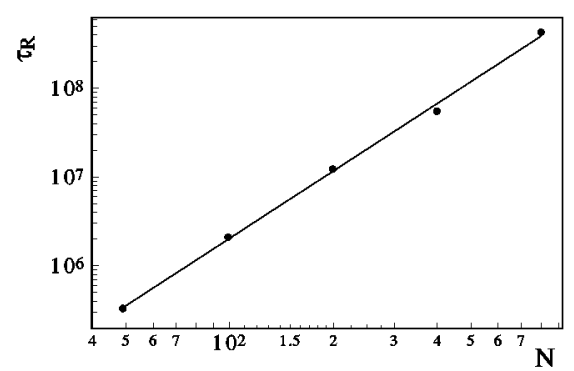

Fig. 3. The longest relaxation times $\tau_{\mathrm{R}}$ versus the length of the chain $N$ at the density $\varphi=0.5$. 
scaling $(N-1)^{3.4}$ for sufficiently long chains $[1,3,4]$. Lower exponents obtained in simulations can be explained by the fact that the density of our melt is only 0.5 ; rough extrapolations towards the concentrated solution $(\varphi=1)$ which is out of simulation capabilities led to the exponents close to 3.4 [5]. Anyway, the short-time dynamic behavior of the chain using the new multibond algorithm is exactly the same as in the classical Monte Carlo algorithm.

The motion of the entire chains (long-time timescale dynamic behavior) can be described and discussed in the term of autocorrelation functions. Center-of-mass autocorrelation function $g_{\mathrm{cm}}(t)$ and single bead autocorrelation function $g(t)$ were obtained and plotted versus time $t$ in Fig. 4 for a system containing chains $N=100$ at $\varphi=0.5$. The behavior of both these functions is qualitatively the same as for isolated chain [14]. The main difference is that for displacements $g_{\mathrm{cm}}(t)<2\left\langle S^{2}\right\rangle$ autocorrelation function scales as $t^{\alpha}$ where $\alpha<1$. The function $g(t)$ also scales with the exponent $\beta<1 / 2$. This behavior, i.e. exponents lower than 1 and $1 / 2$, can be explained by the fact that for short times the motion of a chain is constrained (coupled with the internal relaxation). The shape of both autocorrelation functions does not change with the density and the length of the chain. Self-diffusion coefficients $D$ were determined from the center-of-mass autocorrelation function using modified Einstein's formula

$$
D=g_{\mathrm{cm}}(t) / 6 t+a,
$$

where $a$ is a constant (nonzero value of $a$ is caused by the anomalous diffusion of chains for short times). It was previously shown $[5,14]$ that diffusion coefficient should be determined for the window $g_{\mathrm{cm}}(t)>2\left\langle S^{2}\right\rangle$. In Fig. 5 the self-diffusion coefficient $D$ is plotted against number of polymer segments $N-1$ for chains in a melt at the density $\varphi=0.5$. At these conditions the diffusion coefficient scales with the chain length as $(N-1)^{-1.40 \pm 0.06}$. This value is close to the results given

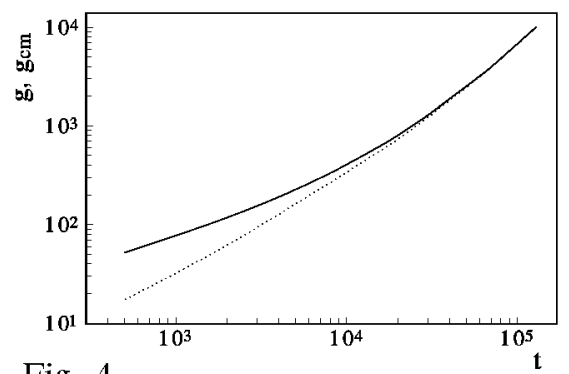

Fig. 4

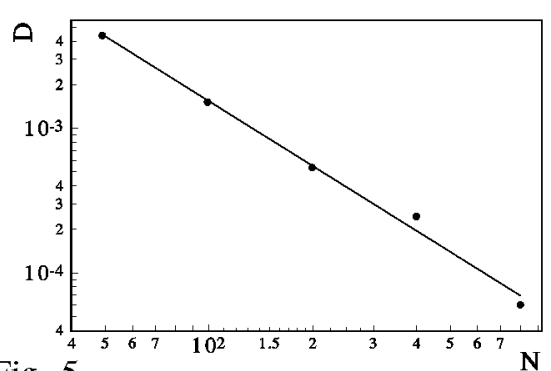

Fig. 5

Fig. 4. Autocorrelation functions $g(t)$ (upper curve) and $g_{\mathrm{cm}}(t)$ (lower curve) vs. time $t$. The case of the chain with $N=100$ beads at the density $\varphi=0.5$.

Fig. 5. Plot of the self-diffusion coefficient $D$ versus the length of the chain $N$ at the density $\varphi=0.5$. 
by the classical algorithm where $D \sim(N-1)^{-1.52}$ [5]. For higher densities it is expected that the exponent should approach $-2[1,3,4]$.

The number of entanglements in chains forming a melt can be estimated from the diffusion coefficient using the Kolinski-Skolnick-Yaris [4, 5] formula, which was based on the Hess treatment [17, 18]:

$$
D=\frac{d_{0}}{N\left(1+N / N_{\mathrm{e}}\right)}
$$

where $N_{e}$ is a number of monomers between entanglements and $d_{0}$ is the effective diffusion coefficient per bead in the absence of chain connectivity. The fitting of this equation for diffusion coefficients obtained by means of the new multibond algorithm for the melt of chains at the density $\varphi=0.5$ gives $N_{e}=121$. This result is very close to that obtained for the same polymer chain model by the classical algorithm where $N_{e}=132$.

The dependence of the self-diffusion coefficient $D$ on the density $\varphi$ of the polymer melt is shown in Fig. 6. One can observe a fast decay of the chain's mobility as the density increases. This is in accordance with other simulation results and theoretical predictions $[3,8]$. The very small values of $D$ for densities over 0.6 suggest that the new algorithm is rather not capable to study the long-time dynamic properties of the system.

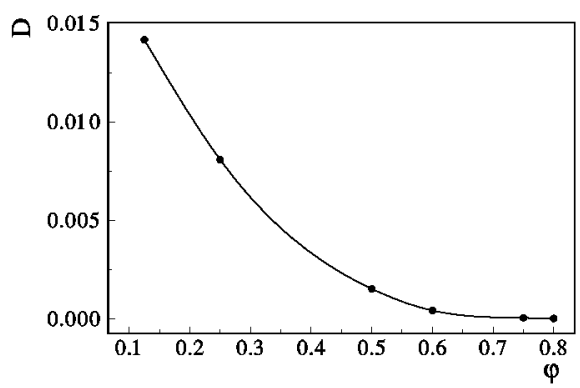

Fig. 6. Plot of the self-diffusion coefficient $D$ versus the density $\varphi$. The case of the chain with $N=100$ beads.

All the results listed above confirm that the new algorithm is capable to reproduce properties not only of an isolated chain [15] but also of dense polymer melts. Moreover, it allows one to determine some properties concerning the nature of chain entanglements which was impossible using the classical algorithm. This is a very important problem as these entanglements determine viscoelastic properties of the polymeric system and no experimental data on entanglements are available.

One has to remember that in the new multibond algorithm there is a possibility of crossing other chains during the attempt of chain's motion [15]. By turning off the procedure that rejects such cases we can therefore remove some of these 
obstacles from the model. These obstacles are apparently related to chains' entanglements that are formed as knots, as well as entanglements formed by looping around a chain. Now we can determine the differences in the chain motion between cases where obstacles are present and suppressed. Therefore, the new algorithm has the ability to determine some properties of entanglements directly from the simulation. During the simulation run all the cases of attempted moves hindered by the above obstacles were analyzed and the number of the unsuccessful moves for each bead of the chain was recorded. Figure 7 shows the plot of the distribution of number of unsuccessful moves $f$ attempted by a given bead (its number is given on $x$-axis). The plot shows that the chain ends have executed more unsuccessful moves than the fragments located in the center of the polymer molecule. It corresponds well with the Rouse-like motion of the chain [3]. Interesting fluctuations of the curve are observed - it shows that even neighboring fragments of the chain exhibit significant differences in their mobility.

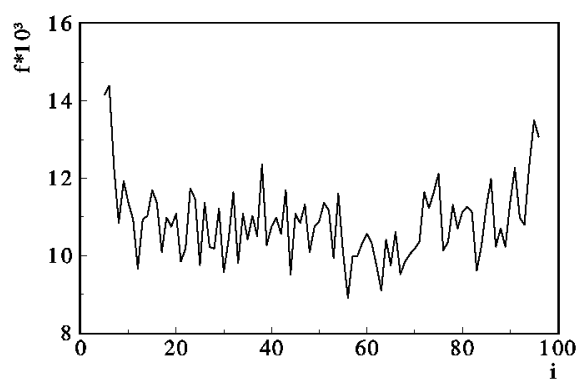

Fig. 7. The distributions of obstacles along the number of chain's bead (see text for details). The case of density $\varphi=0.125$ and $N=100$.

The results described above strongly suggest that the multibond algorithm is capable to reproduce the static and dynamic properties not only for isolated chains but also for dense polymer melts. All the results concerning the polymer size, the process of its internal relaxation, and self-diffusion are the same as obtained by classical Monte Carlo simulation algorithm. The multibond algorithm gives us some insight into the nature of chains entanglements which is very important due to their influence on viscoelastic properties of polymeric systems.

\section{References}

[1] T.P. Lodge, N.A. Rotstein, S. Prager, Adv. Chem. Phys. 79, 1 (1990).

[2] P.G. de Gennes, Scaling Concepts in Polymer Physics, Cornell University Press, Ithaca (NY) 1979.

[3] M. Doi, S.F. Edwards, The Theory of Polymer Dynamics, Oxford Univ. Press, Oxford 1986. 
[4] A. Kolinski, J. Skolnick, Adv. Chem. Phys. 78, 223 (1990).

[5] A. Koliński, J. Skolnick, R. Yaris, J. Chem. Phys. 86, 7164 (1987).

[6] K. Kremer, G. S. Grest, J. Chem. Phys. 92, 5057 (1990).

[7] M. Putz, K. Kremer, G. Grest, Europhys. Lett. 49, 735 (2000).

[8] H. Fuijta, Polymer Solutions, Elsevier, Amsterdam 1990.

[9] C.C. Sorensen, J. Kovac, Macromolecules 24, 3883 (1991).

[10] A. Sikorski, Macromol. Theory Simul. 9, 564 (2000).

[11] A. Kolinski, M. Vieth, A. Sikorski, Acta Phys. Pol. A 79, 601 (1991).

[12] A. Sikorski, Makromol. Chem., Theory Simul. 2, 309 (1993).

[13] A. Sikorski, A. Kolinski, J. Skolnick, Macromol. Theory Simul. 4, 715 (1994).

[14] A. Sikorski, P. Romiszowski, J. Chem. Phys. 104, 8703 (1996).

[15] P. Romiszowski, A. Sikorski, Acta Phys. Pol. A 96, 691 (1999).

[16] P.E. Rouse, J. Chem. Phys. 21, 1272 (1953).

[17] W. Hess, Macromolecules 20, 2587 (1987).

[18] W. Hess, Macromolecules 19, 1395 (1986). 